\title{
新型吡嗪氨基腿类化合物的合成及除草活性
}

\author{
马青森刘幸海翁建全李永曙 \\ 张旻张向阳谭成侠* \\ (浙江工业大学化学工程与材料学院 杭州 310014)
}

\begin{abstract}
摘要 为寻求具有较高生物活性的农药新品种, 根据亚活性拼接原理, 将吡嗪环和氨基腿结构进行亚活性结构拼接, 设计并合成了 26 个未见文献报道的吡嗪氨基腿类化合物，其结构均经 ${ }^{1} \mathrm{H}$ NMR, FTIR 和 MS 确证, 同时对化合物进行 了初步除草活性测试. 活性测试结果表明, 在浓度为 150 gai / ha 时, $\mathbf{4 m}$ 对反枝茡、鳢肠的芽后抑制率达到 $80 \%$.

关键词 吡嗪; 氨基腿; 合成; 除草活性
\end{abstract}

\section{Synthesis and Herbicidal Activity of New Pyrazine Derivative}

\author{
Ma, Qingsen Liu, Xinghai Weng, Jianquan Li, Yongshu \\ Zhang, Min Zhang, Xiangyang Tan, Chengxia* \\ (College of Chemical Engineering and Materials Science, Zhejiang University of Technology, Hangzhou 310014)
}

\begin{abstract}
To find new pesticidal lead compounds with high bioactivity, 26 new pyrazines emicarbazide derivatives were designed and synthesized by combining with the pyrazine ring and semicarbazide. Their structures were confirmed by ${ }^{1} \mathrm{H}$ NMR, FTIR and MS techniques. The herbicidal activities of these compounds were evaluated. The bioassay results showed that the post-emergence inhibitory rate of $\mathbf{4 m}$ reached $80 \%$ on Amaranthus retroflexus and Eclipta prostrata at 150 gai/ha.

Keywords pyrazine; semicarbazide; synthesis; herbicidal activity
\end{abstract}

杂环农药以其多变灵活的结构和高活性等特点, 已 成为化学农药的主要研究方向. 而含氮杂环在农药研究 中占有非常重要的地位 ${ }^{[1 \sim 4]}$, 其中吡嗪类衍生物是一种 具有广泛生物活性的含氮杂环化合物 ${ }^{[5]}$, 是重要的食品 香料，同时也是合成药物、农药、织物整理液、抗静电 剂等的重要中间体. 早在 1879 年人们就从甜菜发酵的 杂醇油中发现并分离了四甲基吡嗪, 并于 1888 年首次 人工合成了吡嗪, 当时人们就已经开始注意到了此类化 合物在医药上的重要价值, 最早应用于抗结核药物, 如 吡嗪酰胺(Pyrazinamide, PZA), 又名氨甲酰基吡嗪 ${ }^{[6]}$. 此外, 吡嗪酰胺还可以作为抗癌药, 它对艾尔利希腹水 肿瘤细胞有显著抑制作用，可有效抑制肿瘤细胞有丝分 裂, 减少细胞数量, 使肿瘤细胞从福水中消失 ${ }^{[7]}$. 吡嗪 类衍生物因具有杀虫杀螨 ${ }^{[8-11]}$ 、除草 ${ }^{[8,10,12]}$ 、杀菌、植物 生长调节 ${ }^{[13]}$ 等活性, 成为许多农药工作者的研究热点.
早在 1957 年就开发出含吡嗪环的具有非内吸性的杀螨 杀菌剂克杀螨(thiquinox)、几丁质抑制剂溙虫嘱、二氯 溙虫脲等具有代表性的商品化杀虫剂品种. 另外, 杀菌 剂叶枯净 (phenazine oxide) 以及除草剂禾草克 (quizalofopethyl)等也是含吡溙环的代表性品种, 经过几 十年的开发研究，目前已发现了大量的吡嗪类衍生物在 农药杀菌剂 ${ }^{[14]}$ 、杀虫剂 ${ }^{[15]}$ 和除草剂 ${ }^{[16]}$ 方面有着良好应 用. 为寻求具有较高生物活性的农药新品种, 本文将取 代芳基异氰酸酯与吡嗪甲酰肼反应，设计并合成了一系 列吡溙氨基腿类化合物, 其结构均经 ${ }^{1} \mathrm{H}$ NMR, FTIR 和 MS 确证. 同时对所有的化合物进行了除草初步活性测 试. 活性测试结果表明，部分化合物对反枝苋、鳢肠的 芽后及狗尾草芽前有较好的除草活性. 合成路线见 Scheme 1, 化合物列表见表 1 .

\footnotetext{
*E-mail: tanchengxia@zjut.edu.cn

Received February 27, 2013; revised April 2, 2013; published online April 12, 2013.

Project supported by the National Key Technologies R\&D Program (No. 2011BAE06B03-01), and the Key Innovation Team of Science and Technology in Zhejiang Province (No. 2010R50018).

“十二五” 国家科技支撑计划(No. 2011BAE06B03-01)、浙江省创新团队(No. 2010R50018)资助项目.
} 
$\mathrm{ArNH}_{2} \stackrel{\mathrm{BTC}, \mathrm{DCM}}{\longrightarrow}$ ArNCO<smiles>COC(=O)c1ncc(OCCNN)c(N)n1</smiles>

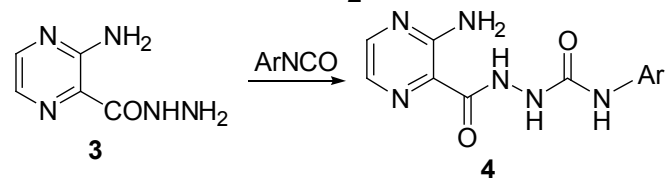

Scheme 1

表 1 化合物 $\mathbf{4 a} \sim \mathbf{4 z}$ 一览表

Table 1 Products of $\mathbf{4 a} \sim \mathbf{4 z}$

\begin{tabular}{cl||cl}
\hline Compd. & \multicolumn{1}{c||}{$\mathrm{Ar}$} & Compd. & \multicolumn{1}{c}{$\mathrm{Ar}$} \\
\hline $\mathbf{4 a}$ & $4-\mathrm{MeO}_{2} \mathrm{CC}_{6} \mathrm{H}_{4}$ & $\mathbf{4 n}$ & $2-\mathrm{FC}_{6} \mathrm{H}_{4}$ \\
$\mathbf{4 b}$ & $2-\mathrm{F}-5-\mathrm{CF}_{3} \mathrm{C}_{6} \mathrm{H}_{3}$ & $\mathbf{4 o}$ & $4-\mathrm{CF}_{3} \mathrm{OC}_{6} \mathrm{H}_{4}$ \\
$\mathbf{4 c}$ & $2-\mathrm{Me}-3-\mathrm{CF}_{3} \mathrm{C}_{6} \mathrm{H}_{3}$ & $\mathbf{4 p}$ & $3-\mathrm{CHF}_{2} \mathrm{OC}_{6} \mathrm{H}_{4}$ \\
$\mathbf{4 d}$ & $2,4,5-\mathrm{F}_{3} \mathrm{C}_{6} \mathrm{H}_{2}$ & $\mathbf{4 q}$ & $2,5-\mathrm{Me}_{2} \mathrm{C}_{6} \mathrm{H}_{3}$ \\
$\mathbf{4 e}$ & $4-\mathrm{EtOC}_{6} \mathrm{H}_{4}$ & $\mathbf{4 r}$ & $2,4-\mathrm{Cl}_{2} \mathrm{C}_{6} \mathrm{H}_{3}$ \\
$\mathbf{4 f}$ & $4-\mathrm{PhOC}_{6} \mathrm{H}_{4}$ & $\mathbf{4 s}$ & $3,4-\mathrm{F}_{2} \mathrm{C}_{6} \mathrm{H}_{3}$ \\
$\mathbf{4 g}$ & $2,3-\mathrm{Me}_{2} \mathrm{C}_{6} \mathrm{H}_{3}$ & $\mathbf{4 t}$ & $2,6-\mathrm{Me}_{2} \mathrm{C}_{6} \mathrm{H}_{3}$ \\
$\mathbf{4 h}$ & $2-\mathrm{CF}_{3} \mathrm{OC}_{6} \mathrm{H}_{4}$ & $\mathbf{4 u}$ & $3,5-\mathrm{Me}_{2} \mathrm{C}_{6} \mathrm{H}_{3}$ \\
$\mathbf{4 i}$ & $2,4-\mathrm{F}_{2} \mathrm{C}_{6} \mathrm{H}_{3}$ & $\mathbf{4 v}$ & $2,4-\mathrm{Me}_{2} \mathrm{C}_{6} \mathrm{H}_{3}$ \\
$\mathbf{4 j}$ & $4-\left(\mathrm{CF}_{3}\right)_{2} \mathrm{CFC}_{6} \mathrm{H}_{4}$ & $\mathbf{4 w}$ & $2,6-\mathrm{Et}_{2} \mathrm{C}_{6} \mathrm{H}_{3}$ \\
$\mathbf{4 k}$ & $4-\mathrm{Me}_{3} \mathrm{CC}_{6} \mathrm{H}_{4}$ & $\mathbf{4 x}$ & $2-\mathrm{CN}_{4}-\mathrm{ClC}_{6} \mathrm{H}_{3}$ \\
$\mathbf{4 l}$ & $3-\mathrm{CF}_{3} \mathrm{C}_{6} \mathrm{H}_{4}$ & $\mathbf{4 y}$ & $3,4-\mathrm{Cl}_{2} \mathrm{C}_{6} \mathrm{H}_{3}$ \\
$\mathbf{4 m}$ & $3-\mathrm{CF}_{3} \mathrm{OC}_{6} \mathrm{H}_{4}$ & $\mathbf{4 z}$ & $3,4-\mathrm{Me}_{2} \mathrm{C}_{6} \mathrm{H}_{3}$ \\
\hline
\end{tabular}

\section{1 结果与讨论}

\section{1 合成及波谱数据}

异氧酸酯采用固体光气(BTC)与取代苯胺反应制 备, 为避免高温下发生聚合, 产生大量的副产物, 因此 在实验过程中, 采用低温滴加 BTC, 待稳定后, 升温至 $70{ }^{\circ} \mathrm{C}$ 反应; 这样有利于提高反应的稳定性和产率. 另
外, 由于异氰酸酯本身不稳定, 因此在合成最终目标产 物的时候都是采用现用现配.

在合成中间体 3-氨基吡嗪-2-甲酰肼的酯化反应中, 当浓硫酸滴加结束，需快速升温至回流反应，尽量减少 副产物的生成.

目标化合物合成时，取代异氰酸酯中若含有吸电子 基团如硝基时，反应收率会有所下降，并且反应时间需 延长至 $8 \mathrm{~h}$ 以上, 因为存在吸电子基团时, 造成基团电 子云密度下降, 不易与吡嗪环上的酰肼反应.

在目标化合物 $\mathbf{4 a} \sim \mathbf{4 z}$ 中存在三个 $\mathrm{NH}$ 基团，化合物 结构式中的 $\mathrm{a}-\mathrm{H}$ 和 $\mathrm{b}-\mathrm{H}$ 分别易与两个羰基氧形成氢键 (如图 2 所示), 使得 $\mathrm{a}-\mathrm{H}$ 和 b-H 在核磁共振谱中的化学 位移出现在低场. 可从核磁共振谱图得到确证, 这三种 $\mathrm{N}-\mathrm{H}$ 的化学位移均出现低场, 大小顺序如下: $\delta\left(\mathrm{H}_{\mathrm{c}}\right)$ $(10.10 \sim 10.41)>\delta\left(\mathrm{H}_{\mathrm{a}}\right)(8.15 \sim 9.24)>\delta\left(\mathrm{H}_{\mathrm{b}}\right)(8.00 \sim 9.20)$, 其中 $\mathrm{H}_{\mathrm{a}}$ 和 $\mathrm{H}_{\mathrm{b}}$ 的位置较难归属, 但是 $\mathrm{Ha}$ 的化学位移变化 比 $\mathrm{H}_{\mathrm{b}}$ 的大, 所以三类氮氢的出峰位置有如上的关系 ${ }^{[17]}$.

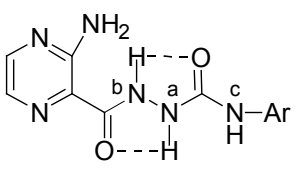

图 2 化合物氢键结构

Figure 2 Hydrogen bond structure of compounds

\section{2 生物活性}

标题化合物委托国家南方农药创制中心浙江基地 生测部测定了对稗草(Echinochloa crusgalli)、狗尾草 (Setaira viridis)、马唐(Digitaria sanguinalis)、芥菜/苟麻 (Brassica juncealAbutilon theophrasti)、反枝苋 (Amaranthus retroflexus)、鳢肠(Eclipta prostrata)的植株 生长抑制, 测定结果列于表 2 . 由表 2 可知, 在浓度为 150 gai /ha 时，化合物 $\mathbf{4 m}$ 对反枝苋、鳢肠的芽后抑制率

表 2 化合物的除草活性(150 gai/ha)

Table 2 Herbicidal activity of compounds (150 gai/ha)

\begin{tabular}{|c|c|c|c|c|c|c|c|c|c|c|c|c|}
\hline \multirow{2}{*}{ 化合物 } & \multicolumn{2}{|c|}{ 稗草 } & \multicolumn{2}{|c|}{ 狗尾草 } & \multicolumn{2}{|c|}{ 马唐 } & \multicolumn{2}{|c|}{ 芥菜/苛麻 } & \multicolumn{2}{|c|}{ 反枝苋 } & \multicolumn{2}{|c|}{ 鳢肠 } \\
\hline & 芽前 & 芽后 & 芽前 & 芽后 & 芽前 & 芽后 & 芽前 & 芽后 & 芽前 & 芽后 & 芽前 & 芽后 \\
\hline $4 c$ & 0 & 0 & 0 & 0 & 0 & 0 & 0 & 0 & 0 & 30 & 0 & 0 \\
\hline $4 e$ & 0 & 0 & 0 & 0 & 0 & 0 & 30 & 0 & 0 & 0 & 30 & 0 \\
\hline $4 g$ & 30 & 0 & 20 & 0 & 20 & 0 & 0 & 0 & 0 & 0 & 0 & 0 \\
\hline $4 k$ & 0 & 0 & 0 & 0 & 0 & 0 & 0 & 0 & 50 & 0 & 20 & 0 \\
\hline $4 m$ & 0 & 0 & 0 & 0 & 0 & 0 & 0 & 0 & 0 & 80 & 0 & 80 \\
\hline 40 & 0 & 0 & 0 & 0 & 0 & 0 & 0 & 0 & 0 & 50 & 0 & 0 \\
\hline $4 q$ & 20 & 0 & 20 & 0 & 20 & 0 & 0 & 0 & 0 & 0 & 0 & 0 \\
\hline $4 r$ & 0 & 0 & 0 & 0 & 0 & 0 & 0 & 0 & 0 & 20 & 0 & 30 \\
\hline $4 s$ & 0 & 0 & 0 & 0 & 0 & 0 & 0 & 0 & 50 & 0 & 0 & 0 \\
\hline $4 t$ & 20 & 0 & 10 & 0 & 20 & 0 & 0 & 0 & 0 & 50 & 0 & 0 \\
\hline $4 u$ & 40 & 0 & 50 & 0 & 0 & 0 & 0 & 0 & 0 & 0 & 0 & 0 \\
\hline $4 w$ & 0 & 0 & 0 & 0 & 0 & 0 & 0 & 0 & 0 & 0 & 50 & 0 \\
\hline
\end{tabular}


为 $80 \%$. 化合物 $\mathbf{4 o}, \mathbf{4 t}$ 对反枝苋的芽后抑制率为 $50 \%$. $4 \mathbf{u}$ 对狗尾草的芽前抑制率为 $50 \%$ ，对稗草的芽前抑制率为 $40 \%$.

\section{2 结论}

本文以 3-氨基吡嗪-2-羧酸为起始原料，通过酯化、 与水合肼反应得到了 3-氨基吡嗪-2-甲酰肼, 进一步与 合成的取代的异氧酸酯反应, 得到了一系列 26 个未见 报道的吡嗪氨基脲类化合物. 并对该系列化合物测试了 熔点, 通过 ${ }^{1} \mathrm{H} N M R, M S$ 和 IR 进行了结构表征. 部分化 合物对反枝苋、鳢肠的芽后抑制率和狗尾草、稗草芽前 抑制率较高. 其中在浓度为 $150 \mathrm{gai} / \mathrm{ha}$ 时, 化合物 $\mathbf{4 m}$ 对 反枝苋、鳢肠的芽后抑制率达到 $80 \%$. 通过构效关系分 析, 当苯环结构中 $\mathrm{CF}_{3} \mathrm{O}$ 取代 3 位 $\mathrm{Ph}-\mathrm{H}$ 时, 除草活性较 好, 但 $\mathrm{CF}_{3} \mathrm{O}$ 取代 2 位或 4 位 $\mathrm{Ph}-\mathrm{H}$ 时活性有所降低甚至 失去拟草活性. 可能是由于 $\mathrm{CF}_{3} \mathrm{O}$ 在苯环 2 位或 4 位时 的电子效应使氨基脲结构中的电子云偏移向吡嗪环侧, 从而导致标题化合物分子不能很好与作用酶进行鳌合 而降低或失去除草活性.

\section{3 实验部分}

\section{1 仪器与试剂}

Varian-400 型核磁共振仪(TMS 为内标); Bruker Avance III 500MHZ 全数字化傅立叶超导核磁共振谱仪 (TMS 为内标); Nicolet 6700 傅立叶变换红外光谱仪; LCQ-Advantage 质谱仪(ESI-MS); X-4 型数字显示熔点 测定仪(温度计未校正); 岛津 LC-10ATVP 高效液相色 谱仪; 旋转蒸发器 IKA-RV10. 所用试剂均为分析纯.

\section{2 化合物的合成}

\subsubsection{3-氨基吡嗪-2-甲酰肼的合成}

$500 \mathrm{~mL}$ 三口烧瓶中, 加入 $300 \mathrm{~mL}$ 甲醇, 冰浴下滴 加 $45 \mathrm{~mL}$ 浓硫酸, 加完后, 恢复至室温; 搅拌下, 加入 $30 \mathrm{~g}$ (0.2 mol) 3-氨基吡嗪-2-羧酸(1), 升温至回流, 固体 溶解; 反应 $2 \sim 3 \mathrm{~h}$ 后, 至原料反应完全, 将反应液倒入 $300 \mathrm{~mL}$ 冰水中, 用 $\mathrm{NaHCO}_{3}$ (或者氨水)调节 $\mathrm{pH}=7$, 冷 却结晶; 过滤, 水洗, 烘干, 得棕色粗品 2 , 收率为 $47 \%$. 将粗品用甲醇脱色, 然后析出大量亮黄色晶体, 过滤甲 醇洗涤, 烘干. 得到黄色晶体. m.p. $185 \sim 187{ }^{\circ} \mathrm{C} ;{ }^{1} \mathrm{H}$ NMR (500 MHz, $\left.\mathrm{CDCl}_{3}\right) \delta: 8.22(\mathrm{~d}, J=2.2 \mathrm{~Hz}, 1 \mathrm{H}), 8.01$ (d, $J=2.2 \mathrm{~Hz}, 1 \mathrm{H}), 4.00$ (s, 3H, $\mathrm{CH}_{3}$ ); MS (ESI) $\mathrm{m} / z: 152$ $[\mathrm{M}-\mathrm{H}]^{-}$.

将上步产品投入反应瓶中, 加入水合肼和甲醇, 室 温下搅拌至原料反应完全(约 $9 \mathrm{~h}$ ), 过滤, 甲醇洗, 烘干, 得到浅黄色固体 3, 收率 73.3\%. m.p. 225 $228{ }^{\circ} \mathrm{C} ;{ }^{1} \mathrm{H}$ NMR (500 MHz, $\left.\mathrm{CDCl}_{3}\right) \delta: 8.78\left(\mathrm{~s}, 1 \mathrm{H}, \mathrm{NHNH}_{2}\right), 8.16$ (d,
$J=2.3 \mathrm{~Hz}, 1 \mathrm{H}), 7.79$ (d, $J=2.4 \mathrm{~Hz}, 1 \mathrm{H}), 3.97(\mathrm{~s}, 2 \mathrm{H}$, $\mathrm{NHNH}_{2}$ ); MS (ESI) $m / z: 152[\mathrm{M}-\mathrm{H}]^{-}$.

\subsection{2 取代芳基异氯酸酯的合成}

在 $250 \mathrm{~mL}$ 烧瓶中加入 $150 \mathrm{~mL}$ 二氯甲烷、芳基苯胺, 在冰盐浴条件下冷至 $5{ }^{\circ} \mathrm{C}$ 以下, 把二氯甲烷溶解的固体 光气滴加到烧瓶中. 加完后, 恒温摚拌 $1 \mathrm{~h}$. 开启回流冷 凝器, 保持温度在 $70{ }^{\circ} \mathrm{C}$ 直到反应混合液由浑浊转为透 明为终点. 反应过程中, 尾气用水、碱两级吸收处理. 用 无水 $\mathrm{N}_{2}$ 吹除反应液中过量的 $\mathrm{HCl}$ 和光气, 得到异氧酸 酯二氯甲烷溶液. 常压蒸出溶剂后, 产物均在 $50 \sim 70$ ${ }^{\circ} \mathrm{C} / 200 \mathrm{kPa}$ 下减压蒸馏所得. 大部分产物常温常压下为 无色液体, 少部分为白色晶体.

\subsection{3 目标化合物的合成}

以目标化合物 $4 \mathrm{a}$ 为例: 将 $1.5 \mathrm{~g}(1 \mathrm{mmol}) 3$-氨基吡 嗪-2-甲酰朋(3)溶解到 $20 \mathrm{~mL}$ 的氯仿溶液中, 加入 1 $\mathrm{mmol}$ 的 4-乙酸苯酯基异氰酸酯, 室温搅拌反应 $3 \mathrm{~h}$, 反 应毕, 脱溶得到白色固体, 用三氯甲烷和乙酸乙酯混合 溶剂重结晶得到相应的目标产物. 采用同样的方法合成 了化合物 $\mathbf{4 b}$ 到 $\mathbf{4 z}$.

4-[2-(3-氨基吡嗪-2-羰基)酰肼甲酰胺]乙酸苯酯 (4a): 白色晶体, 产率 $91.0 \%$. m.p. $253 \sim 255{ }^{\circ} \mathrm{C} ;{ }^{1} \mathrm{H}$ NMR (DMSO- $\left.d_{6}, 400 \mathrm{MHz}\right) \delta$ : 10.29 (s, 1H, CONHAr), 9.17 (s, $1 \mathrm{H}$, PyrazineCONHNH), 8.30 (s, 1H, PyrazineCONHNH), $8.26(\mathrm{~d}, J=2.0 \mathrm{~Hz}, 1 \mathrm{H}$, Pyrazine-H), 7.86 (d, $J=4.0 \mathrm{~Hz}$, 1H, Pyrazine-H), 7.85 (d, $J=8.8 \mathrm{~Hz}, 2 \mathrm{H}, \mathrm{Ph}-\mathrm{H}) 7.61(\mathrm{~d}$, $J=8.4 \mathrm{~Hz}, 2 \mathrm{H}, \mathrm{Ph}-\mathrm{H}), 7.44$ (s, $\left.2 \mathrm{H}, \mathrm{NH}_{2}\right), 3.81$ (s, $3 \mathrm{H}$, $\mathrm{COOCH}_{3}$ ); IR (KBr) $v: 1675,1598 \mathrm{~cm}^{-1}$; MS (ESI) $\mathrm{m} / z$ : $329.2[\mathrm{M}-\mathrm{H}]^{-}$. Anal. calcd for $\mathrm{C}_{14} \mathrm{H}_{14} \mathrm{~N}_{6} \mathrm{O}_{4}$ : C $50.91, \mathrm{H}$ 4.27, N 25.44; found C 50.73, H 4.36, N 25.52.

2-(3-氨基吡嗪-2-羰基)- $N$-(2-氟-5-三氟甲基苯基)氨 基脲(4b): 黄色晶体, 产率 56.0\%. m.p. $>300{ }^{\circ} \mathrm{C} ;{ }^{1} \mathrm{H}$ NMR (DMSO- $\left.d_{6}, 400 \mathrm{MHz}\right) \delta: 10.37$ (s, 1H, CONHAr), 8.58 (s, 1H, PyrazineCONHNH), 8.44 (s, 1H, PyrazineCONHNH), 8.26 (d, $J=2.3 \mathrm{~Hz}, 1 \mathrm{H}$, Pyrazine-H), 8.19 (d, $J=2.2 \mathrm{~Hz}, 1 \mathrm{H}$, Pyrazine-H), $7.98 \sim 7.85(\mathrm{~m}, 2 \mathrm{H}, \mathrm{Ph}-\mathrm{H})$, $7.51 \sim 7.45$ (m, 1H, Ph-H), 7.40 (s, 2H, NH$)_{2}$; IR (KBr) v: 1683, $1654 \mathrm{~cm}^{-1}$; MS (ESI) $\mathrm{m} / z: 357.2[\mathrm{M}-\mathrm{H}]^{-}$. Anal. calcd for $\mathrm{C}_{13} \mathrm{H}_{10} \mathrm{~F}_{4} \mathrm{~N}_{6} \mathrm{O}_{2}$ : C 43.58, $\mathrm{H} 2.81, \mathrm{~N} 23.46$; found C 43.62, H 2.79, N 23.54.

2-(3-氨基吡嗪-2-羰基)- $N$-(2-甲基-3-三氟甲基苯基) 氨基脲(4c): 白色晶体, 产率 $28.2 \%$. m.p. $278 \sim 280{ }^{\circ} \mathrm{C}$; ${ }^{1} \mathrm{H}$ NMR (DMSO- $\left.d_{6}, 400 \mathrm{MHz}\right) \delta$ : 10.30 (s, $1 \mathrm{H}, \mathrm{CON}-$ HAr), $8.44(\mathrm{~s}, 1 \mathrm{H}$, PyrazineCONHNH$), 8.33(\mathrm{~s}, 1 \mathrm{H}$, PyrazineCONHNH), 8.25 (d, $J=2.2 \mathrm{~Hz}, 1 \mathrm{H}$, Pyrazine-H), $7.86\left(\mathrm{~d}, J=2.3 \mathrm{~Hz}, 1 \mathrm{H}\right.$, Pyrazine-H), $7.80\left(\mathrm{~s}, 2 \mathrm{H}, \mathrm{NH}_{2}\right)$, 
$7.48 \sim 7.31(\mathrm{~m}, 3 \mathrm{H}, \mathrm{Ph}-\mathrm{H}), 2.30\left(\mathrm{~s}, 3 \mathrm{H}, \mathrm{CH}_{3}\right)$; IR $(\mathrm{KBr}) v$ : 1692, $1653 \mathrm{~cm}^{-1}$; MS (ESI) $m / z: 353.2[\mathrm{M}-\mathrm{H}]^{-}$. Anal.

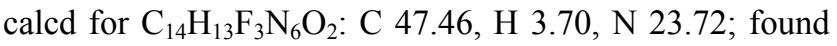
C 47.29, H 4.00, N 23.57.

2-(3-氨基吡嗪-2-羰基)- $N$-(2,4,5-三氟苯基)氨基脲 (4d): 黄色晶体, 产率 55.2\%. m.p. $>300{ }^{\circ} \mathrm{C} ;{ }^{1} \mathrm{H} \mathrm{NMR}$ (DMSO- $\left.d_{6}, 400 \mathrm{MHz}\right) \delta: 10.34$ (s, 1H, CONHAr), 8.81 (s, $1 \mathrm{H}$, PyrazineCONHNH), 8.51 (s, 1H, PyrazineCONHNH), 8.26 (d, $J=2.3 \mathrm{~Hz}, 1 \mathrm{H}$, Pyrazine-H), 8.19 (d, $J=2.4 \mathrm{~Hz}$, 1H, Pyrazine-H), 7.96 (s, 2H, $\left.\mathrm{NH}_{2}\right), 7.86$ (d, $J=2.3 \mathrm{~Hz}$, 1H, Ph-H), 7.59 (dd, $J=18.4,10.8 \mathrm{~Hz}, 1 \mathrm{H}, \mathrm{Ph}-\mathrm{H}$ ); IR (KBr) $v: 1702,1665 \mathrm{~cm}^{-1}$; MS (ESI) $m / z: 325.2[\mathrm{M}-\mathrm{H}]^{-}$. Anal. calcd for $\mathrm{C}_{12} \mathrm{H}_{9} \mathrm{~F}_{3} \mathrm{~N}_{6} \mathrm{O}_{2}$ : C 44.18, H 2.78, N 25.76; found $\mathrm{C} 44.23, \mathrm{H} 2.53, \mathrm{~N} 25.92$.

2-(3-氨基吡嗪-2-羰基)- $N$-(4-乙氧基苯基)氨基脲 (4e): 白色晶体, 产率 82.3\%. m.p. 247 $249{ }^{\circ} \mathrm{C} ;{ }^{1} \mathrm{H}$ NMR (DMSO- $\left.d_{6}, 400 \mathrm{MHz}\right) \delta: 10.18$ (s, 1H, CONHAr), 8.56 (s, 1H, PyrazineCONHNH), 8.25 (d, $J=4.0 \mathrm{~Hz}, 1 \mathrm{H}$, Pyrazine-H), 8.00 (s, 1H, PyrazineCONHNH), 7.86 (d, $J=$ $4.0 \mathrm{~Hz}, 1 \mathrm{H}$, Pyrazine-H), 7.44 (s, 2H, $\left.\mathrm{NH}_{2}\right), 7.33$ (d, $J=$ $8.8 \mathrm{~Hz}, 2 \mathrm{H}, \mathrm{Ph}-2,6-\mathrm{H}), 6.82$ (d, J=8.9 Hz, 2H, Ph-3,5-H), $3.96\left(\mathrm{~d}, J=8.0 \mathrm{~Hz}, 2 \mathrm{H}, \mathrm{CH}_{2} \mathrm{CH}_{3}\right), 1.29$ (t, $J=7.0 \mathrm{~Hz}, 3 \mathrm{H}$, $\mathrm{CH}_{2} \mathrm{CH}_{3}$ ); IR (KBr) v: 1669, $1646 \mathrm{~cm}^{-1}$; MS (ESI) $\mathrm{m} / z$ : $315[\mathrm{M}-\mathrm{H}]^{-}$. Anal. calcd for $\mathrm{C}_{14} \mathrm{H}_{16} \mathrm{~N}_{6} \mathrm{O}_{3}$ : C 53.16, $\mathrm{H}$ 5.10, N 26.57; found C 53.43, H 5.22, N 26.08.

2-(3-氨基吡嗪-2-羰基)- $N$-(4-苯氧基苯基)氨基脲 (4f): 白色晶体，产率 82.4\%. m.p. 207 $210{ }^{\circ} \mathrm{C}$; ${ }^{1} \mathrm{H}$ NMR $\left(\mathrm{DMSO}-d_{6}, 400 \mathrm{MHz}\right) \delta: 10.22$ (s, $\left.1 \mathrm{H}, \mathrm{CONHAr}\right), 8.78$ (s, 1H, PyrazineCONHNH), 8.25 (d, $J=1.9 \mathrm{~Hz}, 1 \mathrm{H}$, Pyrazine-H), 8.10 (s, 1H, PyrazineCONHNH), 7.86 (d, $J=$ $1.9 \mathrm{~Hz}, 1 \mathrm{H}$, Pyrazine-H), 7.47 (t, $J=8.5 \mathrm{~Hz}, 4 \mathrm{H}, \mathrm{CONH}-$ $\mathrm{Ph}-\mathrm{H}$ ), 7.35 (dd, $\left.J=11.6,4.3 \mathrm{~Hz}, 2 \mathrm{H}, \mathrm{NH}_{2}\right), 7.08$ (t, $J=7.4$ $\mathrm{Hz}, 1 \mathrm{H}, \mathrm{OPh}-\mathrm{H}), 6.95$ (d, J=8.8 Hz, 4H, OPh-H); IR (KBr) $v$ : 1705, $1635 \mathrm{~cm}^{-1}$; MS (ESI) $\mathrm{m} / z: 363[\mathrm{M}-\mathrm{H}]^{-}$. Anal. calcd for $\mathrm{C}_{18} \mathrm{H}_{16} \mathrm{~N}_{6} \mathrm{O}_{3}$ : C 59.34, $\mathrm{H}$ 4.43, N 23.07; found C 59.16, H 4.26, N 22.92.

2-(3-氨基吡嗪-2-羰基)- $N$-(2,3-二甲基苯基)氨基脲 (4g): 白色晶体, 产率 83.3\%. m.p. 248 $252{ }^{\circ} \mathrm{C} ;{ }^{1} \mathrm{H}$ NMR (DMSO- $\left.d_{6}, 400 \mathrm{MHz}\right) \delta: 10.22$ (s, 1H, CONHAr), 8.25 (d, $\left.J=2.1 \mathrm{~Hz}, 2 \mathrm{H}, \mathrm{NH}_{2}\right), 8.04$ (s, 1H PyrazineCONHNH), 7.86 (d, $J=2.0 \mathrm{~Hz}, 1 \mathrm{H}$, Pyrazine-H), 7.45 (s, 1H, PyrazineCONHNH), 7.35 (d, $J=7.5 \mathrm{~Hz}, 1 \mathrm{H}$, Pyrazine-H), 7.01 (t, $J=7.8 \mathrm{~Hz}, 1 \mathrm{H}, \mathrm{Ph}-\mathrm{H}), 6.91$ (d, $J=7.5 \mathrm{~Hz}, 2 \mathrm{H}, \mathrm{Ph}-\mathrm{H})$, 2.24 (s, 3H, Ph-3-CH $), 2.10$ (s, 3H, Ph-2- $\mathrm{CH}_{3}$ ); IR (KBr) $v: 1683,1619 \mathrm{~cm}^{-1}$; MS (ESI) $m / z: 299[\mathrm{M}-\mathrm{H}]^{-}$.
Anal. calcd for $\mathrm{C}_{14} \mathrm{H}_{16} \mathrm{~N}_{6} \mathrm{O}_{2}$ : C 55.99, H 5.37, N 27.98; found C 56.13, H 5.39, N 27.82 .

2-(3-氨基吡嗪-2-羰基)- $N$-(2-三氟甲氧基苯基)氨基 脲(4h): 黄色晶体, 产率 50.6\%. m.p. 208 211 ${ }^{\circ} \mathrm{C} ;{ }^{1} \mathrm{H}$ NMR (DMSO- $\left.d_{6}, 400 \mathrm{MHz}\right) \delta: 10.35$ (s, 1H, CONHAr), 8.82 (s, 1H, PyrazineCONHNH), 8.49 (s, 1H, PyrazineCONHNH), 8.26 (d, $J=2.2 \mathrm{~Hz}, 1 \mathrm{H}$, Pyrazine-H), 8.21 8.15 (m, 2H, $\left.\mathrm{NH}_{2}\right), 7.95$ (d, $J=2.3 \mathrm{~Hz}, 1 \mathrm{H}$, Pyrazine-H), 7.90 (d, $J=2.1 \mathrm{~Hz}, 1 \mathrm{H}, \mathrm{Ph}-\mathrm{H}), 7.33$ (dd, $J=17.5,8.4 \mathrm{~Hz}$, 2H, Ph-H), 7.08 (t, $J=7.8 \mathrm{~Hz}, 1 \mathrm{H}, \mathrm{Ph}-\mathrm{H})$; IR (KBr) v: 1696, $1625 \mathrm{~cm}^{-1}$; MS (ESI) $m / z: 355[\mathrm{M}-\mathrm{H}]^{-}$. Anal. calcd for $\mathrm{C}_{13} \mathrm{H}_{11} \mathrm{~F}_{3} \mathrm{~N}_{6} \mathrm{O}_{3}$ : C 43.83, $\mathrm{H} \mathrm{3.11,} \mathrm{N} \mathrm{23.59;} \mathrm{found}$ C 43.68, H 3.09, N 23.63.

2-(3-氨基吡嗪-2-羰基)- $N$-(2,4-二氟苯基)氨基嫝(4i): 黄色晶体，产率 84.4\%. m.p. 265 270 ${ }^{\circ} \mathrm{C} ;{ }^{1} \mathrm{H}$ NMR (DMSO- $\left.d_{6}, 400 \mathrm{MHz}\right) \delta: 10.36$ (s, 1H, CONHAr), 8.63 (s, $1 \mathrm{H}$, PyrazineCONHNH), 8.43 (s, 1H, PyrazineCONHNH), 8.30 (d, $J=2.5 \mathrm{~Hz}, 1 \mathrm{H}$, Pyrazine-H), 7.94 (d, $J=2.5 \mathrm{~Hz}$, 1H, Pyrazine-H), 7.86 (s, 2H, $\left.\mathrm{NH}_{2}\right), 7.29$ (t, $J=10.1 \mathrm{~Hz}$, $1 \mathrm{H}, \mathrm{Ph}-\mathrm{H}), 7.03$ (t, $J=8.4 \mathrm{~Hz}, 1 \mathrm{H}, \mathrm{Ph}-\mathrm{H})$; IR (KBr) v: 1703, $1648 \mathrm{~cm}^{-1}$; MS (ESI) m/z: $307[\mathrm{M}-\mathrm{H}]^{-}$. Anal. calcd for $\mathrm{C}_{12} \mathrm{H}_{10} \mathrm{~F}_{2} \mathrm{~N}_{6} \mathrm{O}_{2}$ : C 46.76, $\mathrm{H}$ 3.27, $\mathrm{N}$ 27.26; found C 46.53, H 3.22, N 27.41.

2-(3-氨基吡嗪-2-羰基)- $N$-[4-(全氟丙烷-2-基)苯基] 氨基脲(4j): 白色晶体，产率 61.4\%. m.p. 145 148 ${ }^{\circ} \mathrm{C}$; ${ }^{1} \mathrm{H}$ NMR (DMSO- $\left.d_{6}, 400 \mathrm{MHz}\right) \delta: 10.37$ (s, 1H, CONHAr), 9.24 (s, 1H, PyrazineCONHNH), 8.35 (s, 1H, PyrazineCONHNH), 8.27 (d, $J=2.2 \mathrm{~Hz}, 1 \mathrm{H}$, Pyrazine-H), $7.88(\mathrm{~d}, J=2.3 \mathrm{~Hz}, 1 \mathrm{H}$, Pyrazine-H), 7.74 (d, $J=7.7 \mathrm{~Hz}$, $\left.2 \mathrm{H}, \mathrm{NH}_{2}\right), 7.60 \sim 7.43(\mathrm{~m}, 4 \mathrm{H}, \mathrm{Ph}-\mathrm{H})$; IR (KBr) v: 1697, $1630 \mathrm{~cm}^{-1}$; MS (ESI) m/z: $439[\mathrm{M}-\mathrm{H}]^{-}$. Anal. calcd for $\mathrm{C}_{15} \mathrm{H}_{11} \mathrm{~F}_{7} \mathrm{~N}_{6} \mathrm{O}_{2}$ : C 40.92, H 2.52, $\mathrm{N}$ 19.09; found $\mathrm{C} 41.07, \mathrm{H}$ 2.50, N 19.13 .

2-(3-氨基吡嗪-2-羰基)- $N$-(4-叔丁基苯基)氨基嫝 (4k): 白色晶体，产率 $82.3 \%$. m.p. $274 \sim 277{ }^{\circ} \mathrm{C} ;{ }^{1} \mathrm{H}$ NMR (DMSO- $\left.d_{6}, 400 \mathrm{MHz}\right) \delta: 10.23(\mathrm{~s}, 1 \mathrm{H}, \mathrm{CONHAr})$, 8.67 (s, 1H, PyrazineCONHNH), 8.22 (d, $J=2.5 \mathrm{~Hz}, 1 \mathrm{H}$, Pyrazine-H), 8.03 (s, 1H, PyrazineCONHNH), 7.83 (d, $J=$ $2.3 \mathrm{~Hz}, 1 \mathrm{H}$, Pyrazine-H), 7.46 (s, 2H, NH 2$), 7.33$ (d, $J=$ $7.9 \mathrm{~Hz}, 2 \mathrm{H}, \mathrm{Ph}-\mathrm{H}), 7.23$ (d, $J=8.0 \mathrm{~Hz}, 2 \mathrm{H}, \mathrm{Ph}-\mathrm{H}), 1.21$ (s, $\left.9 \mathrm{H},\left(\mathrm{CH}_{3}\right)_{3} \mathrm{C}\right)$; IR (KBr) v: 1687, $1624 \mathrm{~cm}^{-1}$; MS (ESI) $m / z: 327[\mathrm{M}-\mathrm{H}]^{-}$. Anal. calcd for $\mathrm{C}_{16} \mathrm{H}_{20} \mathrm{~N}_{6} \mathrm{O}_{2}$ : C 58.52, H 6.14, N 25.59; found C 58.70, H 6.07, N 25.49.

2-(3-氨基吡嗪-2-羰基)- $N$-(3-三氟甲基苯基)氨基脲 (41): 白色晶体，产率 $88.2 \%$. m.p. $238 \sim 242{ }^{\circ} \mathrm{C} ;{ }^{1} \mathrm{H}$ NMR 
(DMSO- $\left.d_{6}, 400 \mathrm{MHz}\right) \delta: 10.36$ (s, 1H, CONHAr), 9.17 (s, $1 \mathrm{H}$, PyrazineCONHNH), 8.37 (s, 1H, PyrazineCONHNH), 8.27 (d, $J=2.5 \mathrm{~Hz}, 1 \mathrm{H}$, Pyrazine-H), 7.88 (s, $2 \mathrm{H}, \mathrm{NH}_{2}$ ), 7.73 (d, $J=2.3 \mathrm{~Hz}, 1 \mathrm{H}$, Pyrazine-H), 7.49 (t, $J=7.5 \mathrm{~Hz}$, 3H, Ph-H), 7.30 (d, $J=7.4 \mathrm{~Hz}, 1 \mathrm{H}, \mathrm{Ph}-\mathrm{H})$; IR (KBr) $v$ : $1675,1615 \mathrm{~cm}^{-1}$; MS (ESI) $m / z: 339[\mathrm{M}-\mathrm{H}]^{-}$. Anal. calcd for $\mathrm{C}_{13} \mathrm{H}_{11} \mathrm{~F}_{3} \mathrm{~N}_{6} \mathrm{O}_{2}$ : C 45.89, H 3.26, N 24.70; found C 46.03, H 3.41, N 24.77.

2-(3-氨基吡嗪-2-羰基)- $N$-(3-三氟甲氧基苯基)氨基 嫝(4m): 白色晶体, 产率 98.3\%. m.p. 239 242 ${ }^{\circ} \mathrm{C} ;{ }^{1} \mathrm{H}$ NMR (DMSO- $\left.d_{6}, 400 \mathrm{MHz}\right) \delta: 10.30$ (s, $1 \mathrm{H}$, CONHAr), 9.09 (s, 1H, PyrazineCONHNH), 8.28 (d, $J=2.5 \mathrm{~Hz}, 1 \mathrm{H}$, Pyrazine-H), 8.23 (s, 1H, PyrazineCONHNH), 7.84 (d, $J=$ $2.3 \mathrm{~Hz}, 1 \mathrm{H}$, Pyrazine-H), 7.63 (s, 1H, Ph-H), 7.46 (s, 2H, $\mathrm{NH}_{2}$ ), 7.34 (d, $\left.J=8.0 \mathrm{~Hz}, 2 \mathrm{H}, \mathrm{Ph}-\mathrm{H}\right), 6.89$ (d, $J=6.6 \mathrm{~Hz}$, $1 \mathrm{H}, \mathrm{Ph}-\mathrm{H}$ ); IR (KBr) $v: 1698,1630 \mathrm{~cm}^{-1}$; MS (ESI) $\mathrm{m} / z$ : $355[\mathrm{M}-\mathrm{H}]^{-}$. Anal. calcd for $\mathrm{C}_{13} \mathrm{H}_{11} \mathrm{~F}_{3} \mathrm{~N}_{6} \mathrm{O}_{3}: \mathrm{C} 43.83, \mathrm{H}$ 3.11, N 23.59; found C 43.68, H 3.04, N 23.64.

2-(3-氨基吡嗪-2-羰基)- $\mathrm{N}$-(2-氟基苯基)氨基脲(4n): 黄色晶体, 产率 96.6\%. m.p. 304 306 ${ }^{\circ} \mathrm{C} ;{ }^{1} \mathrm{H}$ NMR (DMSO- $\left.d_{6}, 400 \mathrm{MHz}\right) \delta: 10.33$ (s, $1 \mathrm{H}, \mathrm{CONHAr}$ ), 8.46 (s, $1 \mathrm{H}$, PyrazineCONHNH), 8.30 (s, 1H, PyrazineCONHNH), $8.22(\mathrm{~d}, J=2.2 \mathrm{~Hz}, 1 \mathrm{H}), 8.16(\mathrm{~d}, J=2.3 \mathrm{~Hz}, 1 \mathrm{H}), 7.94 \sim$ 7.79 (m, 3H, Ph-H), 7.08 (s, 2H, NH${ }_{2}$ ), 6.98 (s, 1H, Ph-H); IR (KBr) $v: 1683,1624 \mathrm{~cm}^{-1}$; MS (ESI) $m / z: 289$ [M$\mathrm{H}]^{-}$. Anal. calcd for $\mathrm{C}_{12} \mathrm{H}_{11} \mathrm{FN}_{6} \mathrm{O}_{2}: \mathrm{C}$ 49.66, H 3.82, N 28.95; found C 49.61, H 3.91, N 29.22.

2-(3-氨基吡嗪-2-羰基)- $N$-(4-三氟甲氧基苯基)氨基 嫝(40): 黄色晶体, 产率 47.8\%. m.p. $319 \sim 322{ }^{\circ} \mathrm{C} ;{ }^{1} \mathrm{H}$ NMR (DMSO- $\left.d_{6}, 400 \mathrm{MHz}\right) \delta: 10.28$ (s, $\left.1 \mathrm{H}, \mathrm{CONHAr}\right)$, 9.12 (s, 1H, PyrazineCONHNH), 8.23 (d, $J=2.2 \mathrm{~Hz}, 1 \mathrm{H})$, $8.16(\mathrm{~d}, J=2.3 \mathrm{~Hz}, 1 \mathrm{H}), 8.16$ (s, $1 \mathrm{H}$, PyrazineCONHNH), $7.85 \sim 7.80\left(\mathrm{~m}, 2 \mathrm{H}, \mathrm{NH}_{2}\right), 7.54(\mathrm{~d}, J=7.6 \mathrm{~Hz}, 2 \mathrm{H}, \mathrm{Ph}-\mathrm{H})$, 7.22 (d, $J=8.7 \mathrm{~Hz}, 2 \mathrm{H}, \mathrm{Ph}-\mathrm{H})$; IR (KBr) $v: 1689,1630$ $\mathrm{cm}^{-1}$; MS (ESI) $\mathrm{m} / z$ : $355[\mathrm{M}-\mathrm{H}]^{-}$. Anal. calcd for $\mathrm{C}_{13} \mathrm{H}_{11} \mathrm{~F}_{3} \mathrm{~N}_{6} \mathrm{O}_{3}: \mathrm{C} 43.83, \mathrm{H} 3.11, \mathrm{~N} 23.59$; found $\mathrm{C} 43.76, \mathrm{H}$ $3.13, \mathrm{~N} 23.64$.

2-(3-氨基吡嗪-2-羰基)- $\mathrm{N}$-(3-二氟甲氧基苯基)氨基 嫝(4p): 白色晶体, 产率 88.7\%. m.p. 223 225 ${ }^{\circ} \mathrm{C} ;{ }^{1} \mathrm{H}$ NMR (DMSO- $\left.d_{6}, 400 \mathrm{MHz}\right) \delta: 10.25$ (s, $\left.1 \mathrm{H}, \mathrm{CONHAr}\right)$, 8.96 (s, 1H, PyrazineCONHNH), 8.25 (d, $J=2.0 \mathrm{~Hz}, 2 \mathrm{H})$, 8.21 (s, 1H, PyrazineCONHNH), 7.87 (d, $J=2.0 \mathrm{~Hz}, 1 \mathrm{H})$, 7.43 (s, 2H, NH${ }_{2}$ ), $\left.7.40 \sim 7.22(\mathrm{~m}, 3 \mathrm{H}, \mathrm{Ph}-\mathrm{H})\right), 7.15(\mathrm{~s}, 1 \mathrm{H}$, $\mathrm{Ph}-\mathrm{H}), 6.76$ (s, 1H, $\mathrm{OCHF}_{2}$ ); IR (KBr) $v: 1684,1647 \mathrm{~cm}^{-1}$; MS (ESI) $m / z: 337[\mathrm{M}-\mathrm{H}]^{-}$. Anal. calcd for $\mathrm{C}_{13} \mathrm{H}_{12} \mathrm{~F}_{2-}$
$\mathrm{N}_{6} \mathrm{O}_{3}$ : C 46.16, H 3.58, N 24.84; found C 46.34, H 3.52, N 24.91.

2-(3-氨基吡嗪-2-羰基)- $\mathrm{N}-(2,5$-二甲基苯基)氨基腿 (4q): 白色晶体, 产率 90.0\%. m.p. $242 \sim 247{ }^{\circ} \mathrm{C} ;{ }^{1} \mathrm{H}$ NMR (DMSO- $\left.d_{6}, 400 \mathrm{MHz}\right) \delta: 10.23$ (s, $\left.1 \mathrm{H}, \mathrm{CONHAr}\right)$, 8.33 (s, 1H, PyrazineCONHNH), 8.25 (d, $J=2.1 \mathrm{~Hz}, 1 \mathrm{H})$, 7.92 (s, 1H, PyrazineCONHNH), 7.86 (d, $J=2.2 \mathrm{~Hz}, 1 \mathrm{H})$, 7.46 (d, $J=13.2 \mathrm{~Hz}, 3 \mathrm{H}, \mathrm{Ph}-\mathrm{H}), 7.03$ (d, $J=7.5 \mathrm{~Hz}, 1 \mathrm{H})$, $6.78(\mathrm{~d}, J=7.5 \mathrm{~Hz}, 1 \mathrm{H}), 2.23\left(\mathrm{~s}, 3 \mathrm{H}, \mathrm{CH}_{3}\right), 2.16(\mathrm{~s}, 3 \mathrm{H}$, $\mathrm{CH}_{3}$ ); IR (KBr) $v$ : 1690, $1642 \mathrm{~cm}^{-1}$; MS (ESI) $m / z: 299$ $[\mathrm{M}-\mathrm{H}]^{-}$. Anal. calcd for $\mathrm{C}_{14} \mathrm{H}_{16} \mathrm{~N}_{6} \mathrm{O}_{2}$ : C 55.99, H 5.37, N 27.98; found C 55.73, H 5.44, N 28.05.

2-(3-氨基吡嗪-2-羰基)- $N$-(2,4-二氯苯基)氨基腿 (4r): 白色晶体, 产率 $88.2 \%$. m.p. $272 \sim 275{ }^{\circ} \mathrm{C} ;{ }^{1} \mathrm{H}$ NMR (DMSO- $\left.d_{6}, 400 \mathrm{MHz}\right) \delta: 10.37$ (s, 1H, CONHAr), 8.86 (s, $1 \mathrm{H}$, PyrazineCONHNH), 8.35 (s, $1 \mathrm{H}$, PyrazineCONHNH), $8.26(\mathrm{~d}, J=2.1 \mathrm{~Hz}, 1 \mathrm{H}), 8.09$ (d, $J=9.0 \mathrm{~Hz}, 1 \mathrm{H}), 7.87$ (d, $J=2.2 \mathrm{~Hz}, 1 \mathrm{H}), 7.60(\mathrm{~d}, J=2.2 \mathrm{~Hz}, 1 \mathrm{H}), 7.49 \sim 7.33(\mathrm{~m}$, $3 \mathrm{H}, \mathrm{Ph}-\mathrm{H}$ ); IR (KBr) v: 1693, $1635 \mathrm{~cm}^{-1}$; MS (ESI) $\mathrm{m} / z$ : $339[\mathrm{M}-\mathrm{H}]^{-}$. Anal. calcd for $\mathrm{C}_{12} \mathrm{H}_{10} \mathrm{Cl}_{2} \mathrm{~N}_{6} \mathrm{O}_{2}: \mathrm{C} 42.25, \mathrm{H}$ 2.95, N 24.63; found C 42.41, H 3.02, N 24.54.

2-(3-氨基吡嗪-2-羰基)- $N$-(3,4-二氟苯基)氨基腿 (4s): 淡黄色晶体, 产率 $71.4 \%$. m.p. $248 \sim 253{ }^{\circ} \mathrm{C} ;{ }^{1} \mathrm{H}$ NMR (DMSO- $\left.d_{6}, 400 \mathrm{MHz}\right) \delta: 10.26(\mathrm{~s}, 1 \mathrm{H}, \mathrm{CONHAr})$, 9.02 (s, 1H, PyrazineCONHNH), 8.27 (d, $J=6.0 \mathrm{~Hz}, 2 \mathrm{H})$, $7.87(\mathrm{~d}, J=2.2 \mathrm{~Hz}, 1 \mathrm{H}), 7.64(\mathrm{dd}, J=13.5,7.7 \mathrm{~Hz}, 1 \mathrm{H})$, 7.45 (s, 1H, PyrazineCONHNH), $7.37 \sim 7.17(\mathrm{~m}, 3 \mathrm{H}$, Ph-H); IR (KBr) $v$ : 1696, $1627 \mathrm{~cm}^{-1}$; MS (ESI) $\mathrm{m} / z: 307$ $[\mathrm{M}-\mathrm{H}]^{-}$. Anal. calcd for $\mathrm{C}_{12} \mathrm{H}_{10} \mathrm{~F}_{2} \mathrm{~N}_{6} \mathrm{O}_{2}$ : C 46.76, H 3.27, N 27.26; found C 46.92, H 3.23, N 27.64.

2-(3-氨基吡嗪-2-羰基)- $N$-(2,6-二甲基苯基)氨基嫝 (4t): 白色晶体, 产率 $93.3 \%$. m.p. $282 \sim 287{ }^{\circ} \mathrm{C} ;{ }^{1} \mathrm{H}$ NMR (DMSO- $\left.d_{6}, 400 \mathrm{MHz}\right) \delta: 10.19$ (s, 1H, CONHAr), 8.23 (d, $J=2.0 \mathrm{~Hz}, 1 \mathrm{H}), 8.04$ (s, 1H, PyrazineCONHNH), 7.93 (s, 1H, PyrazineCONHNH), $7.84(\mathrm{~d}, J=2.1 \mathrm{~Hz}, 1 \mathrm{H}), 7.44$ (s, 2H, $\mathrm{NH}_{2}$ ), 7.02 (s, 3H, Ph-H), 2.19 (s, 6H); IR (KBr) v: 1674, $1634 \mathrm{~cm}^{-1}$; MS (ESI) $m / z: 299[\mathrm{M}-\mathrm{H}]^{-}$. Anal. calcd for $\mathrm{C}_{14} \mathrm{H}_{16} \mathrm{~N}_{6} \mathrm{O}_{2}$ : C 55.99, H 5.37, N 27.98; found C $55.42, \mathrm{H} 5.29, \mathrm{~N} 28.03$.

2-(3-氨基吡嗪-2-羰基)- $N$-(3,5-二甲基苯基)氨基脲 (4u): 白色晶体, 产率 83.3\%. m.p. 251 255 ${ }^{\circ} \mathrm{C} ;{ }^{1} \mathrm{H}$ NMR (DMSO- $\left.d_{6}, 400 \mathrm{MHz}\right) \delta: 10.22$ (s, 1H, CONHAr), 8.57 (s, 1H, PyrazineCONHNH), 8.21 (d, $J=2.0 \mathrm{~Hz}, 1 \mathrm{H})$, 8.03 (s, 1H, PyrazineCONHNH), $7.82(\mathrm{~d}, J=2.1 \mathrm{~Hz}, 1 \mathrm{H})$, 7.44 (s, 2H, NH $\mathrm{N}_{2}$ ), 7.04 (s, 2H, Ph-H), 6.55 (s, 1H, Ph-H), 
2.16 (s, 6H); IR (KBr) $v: 1682,1628 \mathrm{~cm}^{-1}$; MS (ESI) $m / z$ : $299[\mathrm{M}-\mathrm{H}]^{-}$. Anal. calcd for $\mathrm{C}_{14} \mathrm{H}_{16} \mathrm{~N}_{6} \mathrm{O}_{2}$ : C 55.99, $\mathrm{H}$ 5.37, N 27.98; found C 56.20, H 5.32, N 27.79.

2-(3-氨基吡嗪-2-羰基)- $N$-(2,4-二甲基苯基)氨基艮 (4v): 淡黄色晶体, 产率 $86.7 \%$. m.p. $252 \sim 255{ }^{\circ} \mathrm{C} ;{ }^{1} \mathrm{H}$ NMR (DMSO- $\left.d_{6}, 400 \mathrm{MHz}\right) \delta$ : 10.22 (s, 1H, CONHAr), 8.54 (s, 1H, PyrazineCONHNH), 8.22 (d, $J=2.0 \mathrm{~Hz}, 1 \mathrm{H})$, 8.03 (s, 1H, PyrazineCONHNH), 7.85 (d, $J=2.1 \mathrm{~Hz}, 1 \mathrm{H})$, 7.22 (s, 2H, NH$\left.)_{2}\right), 7.04$ (s, 2H, Ph-H), 6.55 (s, 1H, Ph-H), 2.22 (s, 3H, $\left.\mathrm{CH}_{3}\right), 2.17\left(\mathrm{~s}, 3 \mathrm{H}, \mathrm{CH}_{3}\right)$; IR (KBr) v: 1694, $1648 \mathrm{~cm}^{-1}$; MS (ESI) m/z: $299[\mathrm{M}-\mathrm{H}]^{-}$. Anal. calcd for $\mathrm{C}_{14} \mathrm{H}_{16} \mathrm{~N}_{6} \mathrm{O}_{2}$ : C 55.99, H 5.37, N 27.98; found C 56.08, H $5.40, \mathrm{~N} 27.85$.

2-(3-氨基吡嗪-2-羰基)- $N$-(2,6-二乙基苯基)氨基脲 (4w): 白色晶体, 产率 85.4\%. m.p. 271 274 ${ }^{\circ} \mathrm{C} ;{ }^{1} \mathrm{H}$ NMR (DMSO- $\left.d_{6}, 400 \mathrm{MHz}\right) \delta: 10.21$ (s, 1H, CONHAr), 8.19 (s, 1H, PyrazineCONHNH), 8.03 (d, $J=2.0 \mathrm{~Hz}, 1 \mathrm{H})$, 7.89 (s, 1H, PyrazineCONHNH), 7.81 (d, $J=2.1 \mathrm{~Hz}, 1 \mathrm{H})$, 7.45 (s, 2H, $\mathrm{NH}_{2}$ ), 7.05 (dd, $\left.J=14.5,7.5 \mathrm{~Hz}, 3 \mathrm{H}, \mathrm{Ph}-\mathrm{H}\right)$, $2.53\left(\mathrm{dd}, J=14.5,7.5 \mathrm{~Hz}, 4 \mathrm{H},\left(\mathrm{CH}_{2} \mathrm{CH}_{3}\right)_{2}\right), 1.07$ (t, $J=7.0$ $\left.\mathrm{Hz}, 6 \mathrm{H},\left(\mathrm{CH}_{2} \mathrm{CH}_{3}\right)_{2}\right)$; IR (KBr) v: 1687, $1635 \mathrm{~cm}^{-1}$; MS (ESI) $m / z: 327[\mathrm{M}-\mathrm{H}]^{-}$. Anal. calcd for $\mathrm{C}_{16} \mathrm{H}_{20} \mathrm{~N}_{6} \mathrm{O}_{2}: \mathrm{C}$ 58.52, H 6.14, N 25.59; found C 58.29, H 5.99, N 25.62.

2-(3-氨基吡嗪-2-羰基)- $N$-(2-氰基-4-氯苯基)氨基艮 (4x): 白色晶体, 产率 84.6\%. m.p. 244 247 ${ }^{\circ} \mathrm{C} ;{ }^{1} \mathrm{H}$ NMR (DMSO- $\left.d_{6}, 400 \mathrm{MHz}\right) \delta: 10.91$ (s, 1H, CONHAr), 8.33 (s, 1H, PyrazineCONHNH), 8.10 (d, $J=8.7 \mathrm{~Hz}, 1 \mathrm{H}), 7.94$ (s, 1H, PyrazineCONHNH), 7.84 (d, $J=2.1 \mathrm{~Hz}, 1 \mathrm{H}), 7.44$ (s, $2 \mathrm{H}, \mathrm{NH}_{2}$ ), $7.33 \sim 7.16$ (m, 2H, Ph-H), 7.12 (s, 1H, Ph-H); IR (KBr) $v: 1694,1638 \mathrm{~cm}^{-1}$; MS (ESI) $\mathrm{m} / z: 330$ [M$\mathrm{H}]^{-}$. Anal. calcd for $\mathrm{C}_{13} \mathrm{H}_{10} \mathrm{ClN}_{7} \mathrm{O}_{2}: \mathrm{C} 47.07, \mathrm{H} 3.04, \mathrm{~N}$ 29.56; found C 47.13, H 3.09, N 29.98.

2-(3-氨基吡嗪-2-羰基)- $N$-(3,4-二氯苯基)氨基脲 (4y): 淡黄晶体, 产率 64.7\%. m.p. 261 264 ${ }^{\circ} \mathrm{C} ;{ }^{1} \mathrm{H}$ NMR (DMSO- $\left.d_{6}, 400 \mathrm{MHz}\right) \delta$ : 10.29 (s, 1H, CONHAr), 9.07 (s, $1 \mathrm{H}$, PyrazineCONHNH), 8.35 (s, 1H, PyrazineCONHNH), 8.25 (d, $J=2.1 \mathrm{~Hz}, 1 \mathrm{H}), 7.86$ (d, $J=2.3 \mathrm{~Hz}, 1 \mathrm{H}), 7.44$ (s, $\left.2 \mathrm{H}, \mathrm{NH}_{2}\right), 7.47$ (t, $\left.J=8.7 \mathrm{~Hz}, 3 \mathrm{H}, \mathrm{Ph}-\mathrm{H}\right)$; IR (KBr) $v$ : 1672, $1588 \mathrm{~cm}^{-1}$; MS (ESI) $m / z: 339[\mathrm{M}-\mathrm{H}]^{-}$. Anal. calcd for $\mathrm{C}_{12} \mathrm{H}_{10} \mathrm{Cl}_{2} \mathrm{~N}_{6} \mathrm{O}_{2}$ : C 42.25, $\mathrm{H}$ 2.95, N 24.63; found C 42.37, H 2.91, N 24.58.
2-(3-氨基吡嗪-2-羰基)- $N$-(3,4-二甲基苯基)氨基脲 (4z): 淡黄晶体，产率 50.0\%. m.p. 240 $244{ }^{\circ} \mathrm{C} ;{ }^{1} \mathrm{H}$ NMR (DMSO- $\left.d_{6}, 400 \mathrm{MHz}\right) \delta: 10.19$ (s, 1H, CONHAr), 8.56 (s, 1H, PyrazineCONHNH), 8.25 (d, $J=2.0 \mathrm{~Hz}, 1 \mathrm{H}), 8.02$ (s, 1H, PyrazineCONHNH), 7.86 (d, $J=2.2 \mathrm{~Hz}, 1 \mathrm{H}), 7.44$ (s, $\left.2 \mathrm{H}, \mathrm{NH}_{2}\right), 7.25 \sim 7.15(\mathrm{~m}, 2 \mathrm{H}, \mathrm{Ph}-\mathrm{H}), 6.99$ (d, J=7.9 Hz, 1H, Ph-H), 2.15 (d, $\left.J=9.4 \mathrm{~Hz}, 6 \mathrm{H},\left(\mathrm{CH}_{3}\right)_{2}\right)$; IR (KBr) v: 1705, $1639 \mathrm{~cm}^{-1}$; MS (ESI) m/z: $299[\mathrm{M}-\mathrm{H}]^{-}$. Anal. calcd for $\mathrm{C}_{14} \mathrm{H}_{16} \mathrm{~N}_{6} \mathrm{O}_{2}$ : C 55.99, H 5.37, N 27.98; found $\mathrm{C}$ 56.12, H 5.31, N 27.73.

\section{References}

[1] Liu, X.-H.; Chen, P.-Q.; Wang, B.-L.; Li, Y.-H.; Wang, S.-H.; Li, Z.-M. Bioorg. Med. Chem. Lett. 2007, 17, 3784.

[2] Liu, X.-H.; Zhang, C.-Y.; Guo, W.-C.; Li, Y.-H.; Chen, P.-Q.; Wang, T.; Dong, W. L.; Sun, H. W.; Li, Z. M. J. Enzyme Inhib. Med. Chem. 2009, 24, 545.

[3] Liu, X.-H.; Shi, Y.-X.; Ma, Y.; Zhang, C.-Y.; Dong, W.-L.; Li, P.; Wang, B.-L.; Li, B.-J.; Li, Z.-M. Eur. J. Med. Chem. 2009, 44, 2782.

[4] Liu, X.-F.; Liu, X.-H. Acta Crystallogr., Sect. E 2011, 67, O202 $\mathrm{U} 3145$.

[5] Song, B.-A.; Wu, J. New Heterocyclic Pesticide (Herbicide), Chemical Industry Press, Beijing, 2011. pp. 143 150 (in Chinese). (宋宝安, 吴剑，新杂环农药 (除草剂)，化学工业出版社，北京， 2011. pp. $143 \sim 150$.)

[6] Elsevier Ltd. Tuberculosis 2008, 88(2), 141.

[7] Zhou, L.-Y.; Feng, Y.-Q.; Qu, H.-M. Chem. Prop. Polym. Mater. 2002, 88(4), 4.

[8] Fischer, G. C. US 4742167, 1988 [Chem. Abstr. 1988, 109, 73476].

[9] Merce, B.; Jesus, A.; Jaume, P.; Ramon, C. J. Agric. Food Chem. 2000, 48(1), 83 .

[10] Allison, C. G.; Chambers, R. D. GB 1342598, 1974 [Chem. Abstr. 1974, 80, 133478].

[11] Gulbenk, A. H.; Johnstou, H. US 3746707, 1973 [Chem. Abstr. 1973, 79, 105301].

[12] Johnston, D. B. US 4518599, 1985 [Chem. Abstr. 1985, 103, 87908].

[13] Krumkalns, E. V. EP 109299, 1984 [Chem. Abstr. 1984, 101, 110960].

[14] Allison, C. G.; Chambers, R. D. GB 1342598, 1974 [Chem. Abstr. 1974, 80, 133470].

[15] John, L. M. US 4160834, 1979 [Chem. Abstr. 1979, 91, 140598].

[16] Tumova, L.; Gallova, K.; Rimakova, J. Acta Physiol. Plant. 2005, 27(3B), 357.

[17] Zhang, X.-Y.; Li, Y.-S.; Weng, J.-Q.; Tan, C.-X. Chin. J. Org. Chem. 2011, 31(8), 1295 (in Chinese).

(张向阳, 李永曙, 翁建全, 谭成侠, 有机化学, 2011，31(8), 1295.) 